\title{
Exclusive photoproduction of charmonia in hadronic collisions
}

\author{
Anna Cisek ${ }^{* \dagger}$ \\ University of Rzeszów, PL-35-959 Rzeszów, Poland \\ E-mail: acisek@univ.rzeszow.pl
}

\section{Wolfgang Schäfer}

Institute of Nuclear Physics PAN, PL-31-342 Cracow, Poland

E-mail: Wolfgang.Schafer@ifj.edu.pl

\section{Antoni Szczurek}

University of Rzeszów, PL-35-959 Rzeszów, Poland

Institute of Nuclear Physics PAN, PL-31-342 Cracow, Poland

E-mail: Antoni.Szczurek@ifj.edu.pl

\begin{abstract}
We present results based on our new paper [1]. The amplitude for the $\gamma p \rightarrow V p$ reaction is calculated in a pQCD $k_{T}$-factorization approach. The coresponding total cross section for different unintegrated gluon distributions is calculated and compared with new HERA data for photonproton collisions. We also compare the total cross section for $\gamma p \rightarrow V p$ reaction with recent data extracted by the LHCb collaboration. The amplitude for $\gamma p \rightarrow V p$ is used to predict cross section for exclusive photoproduction of $J / \psi$ and $\psi^{\prime}$ mesons in proton-proton collisions. Both Dirac and Pauli electromagnetic form factors are included in the calculation and results are compared with old results (only Dirac form factor). The effect of Pauli form factor is quantified. Absorption effects are included and their uncertainties are discussed. Different differential distributions e.g. in $J / \psi\left(\psi^{\prime}\right)$ rapidity and transverse momentum are presented and compared with existing experimental data for the Tevatron and LHC energy.
\end{abstract}

XXII. International Workshop on Deep-Inelastic Scattering and Related Subjects, 28 April - 2 May 2014

Warsaw, Poland

\footnotetext{
* Speaker.

${ }^{\dagger}$ partially supported by Polish NCN grant DEC-2011/01/B/ST2/04535
} 


\section{Introduction}

A few years ago in Ref.[2] the exclusive production of $J / \psi$ meson in proton-proton and proton-antiproton collisions was presented. It was shown that the exclusive production of $J / \psi$ on the Tevatron is related to the photon-proton scattering in the similar region of energy as measured at HERA. The formalism proposed in [3] and used in [2] allows to calculate fully differential distributions for the three-body reaction in the broad range of four-dimensional phase space. The formalism proposed in paper [3] allows to test unintegrated gluons distributions (UGDF's). In our cacluclation we used two different wave functions of the vector meson: Gaussian and Coulombic. The experimental data for production of different vector mesons prefer the Gaussian light-cone wave function [3, 4]. Recently also the LHCb collaboration measured rapidity distribution of the $J / \psi[5]$ and $\psi^{\prime}[6]$ mesons and we will compare our calculation to their results.

\section{Photoproduction $\gamma p \rightarrow V p$ at HERA}

The amplitude for the reaction is shown schematically in Fig.1. The full amplitude for this process can be written as:

$$
M\left(W, \Delta^{2}\right)=(i+\rho) \mathfrak{I} m M\left(W, \Delta^{2}=0, Q^{2}=0\right) \exp \left(\frac{-B(W) \Delta^{2}}{2}\right),
$$

where $\rho$ is the ratio of real to imaginary part of the amplitude and $B(W)$ is slope parameter which depends on energy: $B(W)=B_{0}+2 \alpha_{e f f}^{\prime} \log \left(\frac{W^{2}}{W_{0}^{2}}\right)[1,3]$. The imaginary part of the amplitude depends on the unintegrated gluon distibution function (UGDF) $F\left(x_{e f f}, \kappa^{2}\right)$ and on the wave function of the vector meson $\psi_{V}\left(z, k^{2}\right)$ and for $\Delta=0$ and $Q^{2}=0$ is given by the formula [4]:

$$
\begin{gathered}
\mathfrak{I} m M\left(W, \Delta^{2}=0, Q^{2}=0\right)=W^{2} \frac{c_{v} \sqrt{4 \pi \alpha_{e m}}}{4 \pi^{2}} 2 \int_{0}^{1} \frac{d z}{z(1-z)} \int_{0}^{\infty} \pi d k^{2} \psi_{V}\left(z, k^{2}\right) \\
\int_{0}^{\infty} \frac{\pi d \kappa^{2}}{\kappa^{4}} \alpha_{S}\left(q^{2}\right) F\left(x_{e f f}, \kappa^{2}\right)\left(A_{0}\left(z, k^{2}\right) W_{0}\left(k^{2}, \kappa^{2}\right)+A_{1}\left(z, k^{2}\right) W_{1}\left(k^{2}, \kappa^{2}\right)\right),
\end{gathered}
$$

where

$$
\begin{aligned}
A_{0}\left(z, k^{2}\right) & =m_{c}^{2}+\frac{k^{2} m_{c}}{M+2 m_{c}}, \\
A_{1}\left(z, k^{2}\right) & =\left[z^{2}+(1-z)^{2}-(2 z-1)^{2} \frac{m_{c}}{M+2 m_{c}}\right] \frac{k^{2}}{k^{2}+m_{c}^{2}}, \\
W_{0}\left(k^{2}, \kappa^{2}\right) & =\frac{1}{k^{2}+m_{c}^{2}}-\frac{1}{\sqrt{\left(k^{2}-m_{c}^{2}-\kappa^{2}\right)^{2}+4 m_{c}^{2} k^{2}}}, \\
W_{1}\left(k^{2}, \kappa^{2}\right) & =1-\frac{k^{2}+m_{c}^{2}}{2 k^{2}}\left(1+\frac{k^{2}-m_{c}^{2}-\kappa^{2}}{\sqrt{\left(k^{2}-m_{c}^{2}-\kappa^{2}\right)^{2}+4 m_{c}^{2} k^{2}}}\right) .
\end{aligned}
$$

Total cross section can be calculated as:

$$
\sigma(\gamma p \rightarrow J / \psi p)=\frac{1+\rho^{2}}{16 \pi B(W)}\left|\frac{\Im m M\left(W, \Delta^{2}=0, Q^{2}=0\right)}{W^{2}}\right|^{2} .
$$

In our calculation we used two types of the wave functions, Gaussian wave function [4]: 


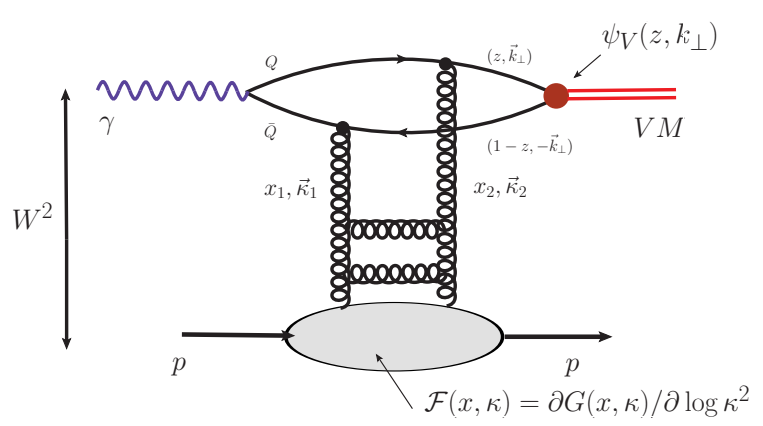

Figure 1: A sketch of the amplitude for exclusive photoproduction $\gamma p \rightarrow V p$

$$
\psi_{1 S}\left(p^{2}\right)=C_{1} \exp \left(-\frac{p^{2} a_{1}^{2}}{2}\right), \psi_{2 S}\left(p^{2}\right)=C_{2}\left(\xi_{0}-p^{2} a_{2}^{2}\right) \exp \left(-\frac{p^{2} a_{2}^{2}}{2}\right)
$$

and Coulomb-like wave function:

$$
\psi_{1 S}\left(p^{2}\right)=\frac{C_{1}}{\sqrt{M}} \frac{1}{\left(1+a_{1}^{2} p^{2}\right)^{2}}, \psi_{2 S}\left(p^{2}\right)=\frac{C_{2}}{\sqrt{M}} \frac{\xi_{0}-a_{2}^{2} p^{2}}{\left(1+a_{2}^{2} p^{2}\right)^{3}} .
$$

The parameters of the wave function are obtained from fitting the decay widths into $e^{+} e^{-}$.
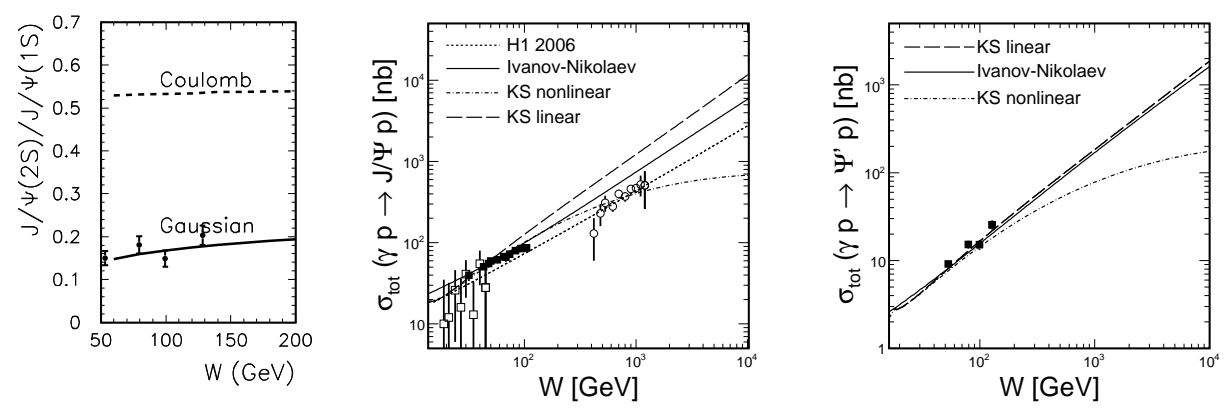

Figure 2: Left panel: Ratio of the first radial excitation state to ground state as a function of energy. Middle panel: Total cross section for the $J / \psi$ meson photoproduction as a function of the energy. Right panel: Total cross section for the $\gamma p \rightarrow \psi^{\prime} p$ as a function of the energy.

In Fig.2 in the left panel we show the ratio of the cross section for the first radial excitation state $\psi^{\prime}$ to the cross section for the ground state $J / \psi$. The ratio of the $2 \mathrm{~S} / 1 \mathrm{~S}$ is strongly dependent on the model of the wave function. The solid line is for the Gaussian wave function and the dashed line is for the Coulomb wave function. For the following calculations we used Gaussian wave function. In the middle panel we present the total cross section for $\gamma p \rightarrow J / \psi p$ and in the right panel for $\gamma p \rightarrow \psi^{\prime} p$. The total cross section is shown as a function of collision energy for the different UGDFs models: Ivanov-Nikolaev (solid), Kutak-Stasto linear (dashed) and Kutak-Stasto nonlinear (dash-dotted). The dotted line (only for $J / \psi$ ) represents a calculation with a simple 
power-like parametrization of the old HERA data [7]. The HERA data points [8] and the LHCb data ponits $[5,6]$ are shown for comparison.

\section{Exclusive photoproduction of $J / \psi$ and $\psi^{\prime}$ meson in $p p$ and $p \bar{p}$ collisions.}
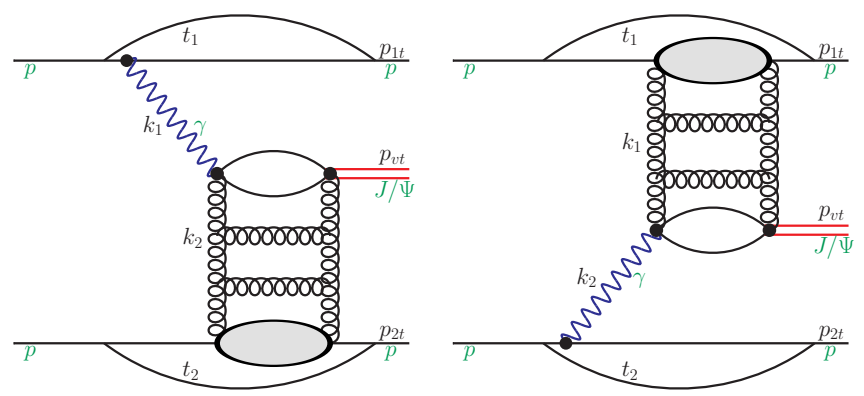

Figure 3: Diagrams representing Born amplitude for the $p p \rightarrow p J / \psi p$ process.
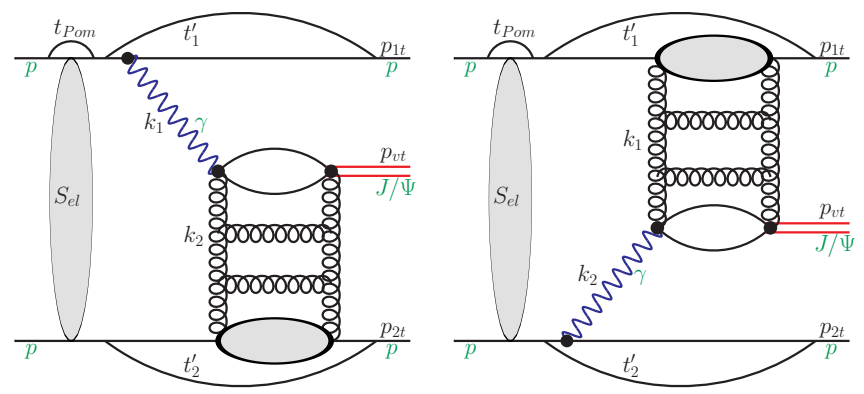

Figure 4: Diagrams representing absorptive corrections considered for the $p p \rightarrow p J / \psi p$ process.

The full amplitude for the $p p \longrightarrow p J / \psi p$ is calculated as:

$$
M_{h_{1} h_{2} \rightarrow h_{1} h_{2} V}^{\lambda_{1} \lambda_{2} \rightarrow \lambda_{1}^{\prime} \lambda_{2}^{\prime} \lambda_{V}}\left(p_{1}, p_{2}\right)=M^{(0)} \underset{h_{1} h_{2} \rightarrow h_{1} h_{2} V}{\lambda_{2} \lambda_{2} \rightarrow \lambda_{1}^{\prime} \lambda_{2}^{\prime} \lambda_{V}}\left(s, s_{1}, s_{2}, t_{1}, t_{2}\right)-\delta M_{h_{1} h_{2} \rightarrow h_{1} h_{2} V}^{\lambda_{1} \lambda_{2} \rightarrow \lambda_{1}^{\prime} \lambda_{2}^{\prime} \lambda_{V}}\left(p_{1}, p_{2}\right) .
$$

The Born amplitude (shown schematically in Fig.3) for the $p p \rightarrow p J / \psi p$ process is given by formula [1]:

$$
\begin{aligned}
& M_{h_{1} h_{2} \rightarrow h_{1} h_{2} V}^{\left(0 \lambda_{2} \lambda_{2} \rightarrow \lambda^{\prime} \lambda_{2}^{\prime} \lambda_{V}\right.}\left(s, s_{1}, s_{2}, t_{1}, t_{2}\right)=M_{\gamma P}+M_{P \gamma} \\
& =<p_{1}^{\prime}, \lambda_{1}^{\prime}\left|J_{\mu}\right| p_{1}, \lambda_{1}>\varepsilon_{\mu}^{*}\left(q_{1}, \lambda_{V}\right) \frac{\sqrt{4 \pi \alpha_{e m}}}{t_{1}} M_{\gamma^{*} h_{2} \rightarrow V h_{2}}^{\lambda_{\gamma^{*}} \lambda_{2} \rightarrow \lambda_{V} \lambda_{2}}\left(s_{2}, t_{2}, Q_{1}^{2}\right) \\
& +<p_{2}^{\prime}, \lambda_{2}^{\prime}\left|J_{\mu}\right| p_{2}, \lambda_{2}>\varepsilon_{\mu}^{*}\left(q_{2}, \lambda_{V}\right) \frac{\sqrt{4 \pi \alpha_{e m}}}{t_{2}} M_{\gamma^{*} h_{1} \rightarrow V h_{1}}^{\lambda_{\gamma^{*}} \lambda_{1} \rightarrow \lambda_{V} \lambda_{2}}\left(s_{1}, t_{1}, Q_{2}^{2}\right) \text {. }
\end{aligned}
$$


The absorptive correction (shown schematically in Fig. 4 as $S_{e l}$ ) to the amplitude can be written as [2]:

$$
\delta M_{h_{1} h_{2} \rightarrow h_{1} h_{2} V}^{\lambda_{1} \lambda_{2} \rightarrow \lambda_{1}^{\prime} \lambda_{2}^{\prime} \lambda_{V}}\left(p_{1}, p_{2}\right)=\int \frac{d^{2} k}{2(2 \pi)^{2}} T(k) M_{{ }_{1} h_{2} \rightarrow h_{1} h_{2} V}^{\lambda_{1} \lambda_{2} \rightarrow \lambda_{1}^{\prime} \lambda_{2}^{\prime} \lambda_{V}}\left(p_{1}-k, p_{2}+k\right)
$$

where

$$
T(k)=\sigma_{t o t}^{p p}(s) \exp \left(-\frac{1}{2} B_{e l} k^{2}\right) .
$$

In practice for the LHC energy $(W=7 \mathrm{TeV})$ we take $B_{e l}=19.89 \mathrm{GeV}^{2}$ and $\sigma_{t o t}^{p p}(s)=98.6$ $\mathrm{mb}[9]$.
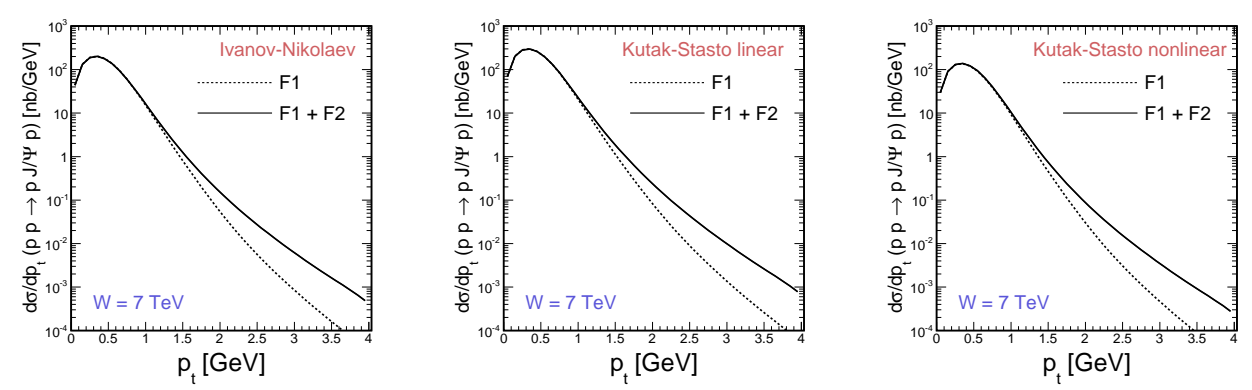

Figure 5: $J / \psi$ transverse momentum distribution calculated with the Born amplitudes for three different UGDFs: Ivanov-Nikolaev (left panel), Kutak-Staśto linear (middle panel) and Kutak-Staśto nonlinear (right panel). The dashed lines include contributions with the Dirac $F_{1}$ electromagnetic form factor and the solid lines include in addition Pauli $F_{2}$ electromagnetic form factor.

Distribution in the transverse momentum of $J / \psi$ meson is presented in Fig.5. The role of Pauli electromagnetic form factor is quantified in [1]. Large effect of the tensor coupling is observed for large transverse momenta. Large transverse momenta are potentially interesting because this can be related to Odderon exchange.

In Fig.6 we shown rapidity distribution for $J / \psi$ (upper plots) and $\psi^{\prime}$ (lower plots) mesons for three different UGDFs models: Ivanov-Nikolaev (left panel), Kutak-Staśto linear (middle panel) and Kutak-Staśto nonlinear (right panel). The absorption damps rapidity distribution by about $30 \%$. We present results with the standard absorption (elastic rescattering) as well as with the absorption increased by factor a 1.4 to simulate inelastic terms. The results with the Kutak-Staśto nonlinear distribution are almost consistent with the recent $\mathrm{LHCb}$ data [6].

\section{Conclusions}

We have calculated the total cross section for $J / \psi$ and $\psi^{\prime}$ mesons photoproduction $\gamma p \rightarrow$ $J / \psi\left(\psi^{\prime}\right) p$. These results depend on the wave function of the vector meson and UGDFs. We compare our results with HERA data. Based on the photoproduction $\gamma p \rightarrow J / \psi\left(\psi^{\prime}\right) p$ amplitudes, we calculated cross sections for exclusive $J / \psi$ and $\psi^{\prime}$ mesons photoproduction in proton-proton collisions. We compare our results with the recent $\mathrm{LHCb}$ data $[5,6]$. In our calculation for hadronic 

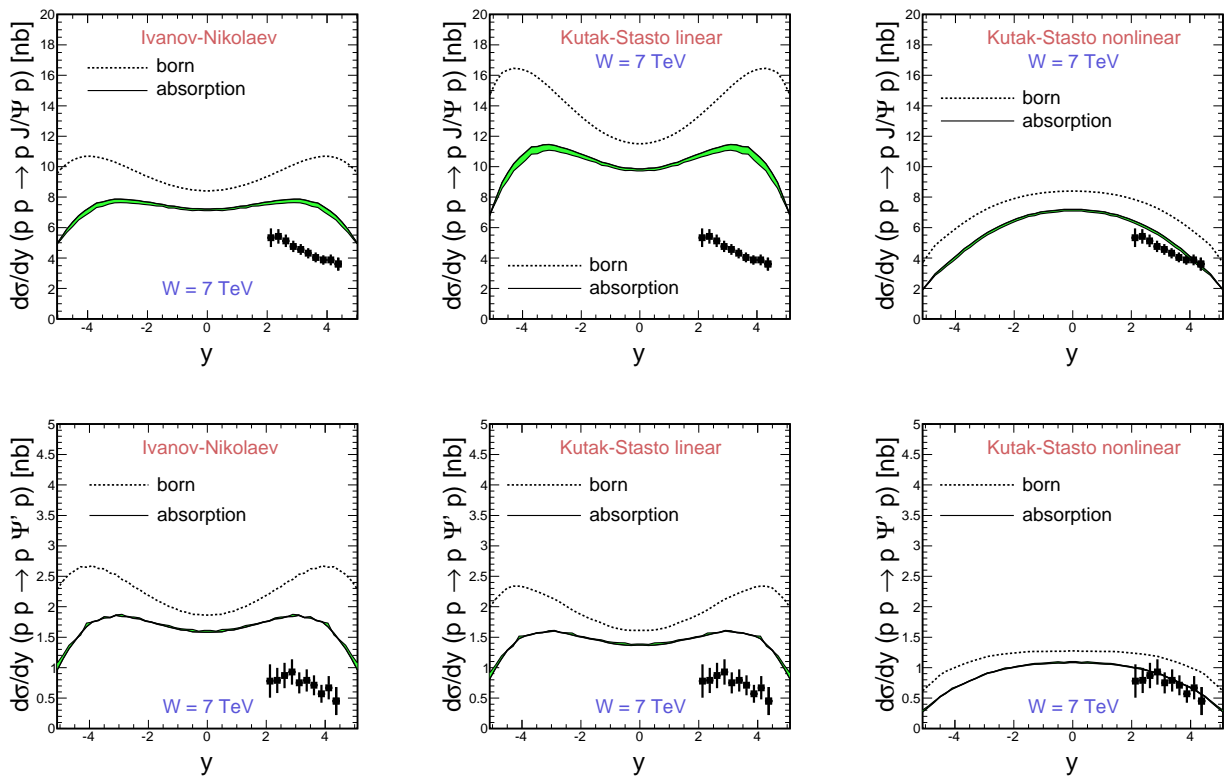

Figure 6: Rapidity distribution of $J / \psi$ (upper plots) and $\psi^{\prime}$ (lower plots) mesons calculated with inclusion of absorption effects (solid lines), compared with the Born result (dashed lines). The new LHCb data points [6] are shown for comparison.

reaction we included the absorption effects. This effect depends on rapidity and transverse momentum of vector mesons. We have taken into account both the coupling of photons via spin-conserving vector coupling with $F_{1}$ Dirac electromagnetic form factor as well as spin-flipping tensor coupling with $F_{2}$ Pauli electromagnetic form factor for the $p p \rightarrow p J / \psi p$ and $p p \rightarrow p \psi^{\prime} p$ reactions. More on the formalism as well as more results for $J / \psi$ and $\psi^{\prime}$ can be found in Ref.[1]

\section{References}

[1] A. Cisek, W. Schäfer and A. Szczurek,arXiv: 1405.2253 [hep-ph].

[2] W. Schäfer and A. Szczurek, Phys. Rev. D76 (2007) 09014.

[3] I. P. Ivanov, N. N. Nikolaev and A. A. Savin, Phys. Part. Nucl. 37 (2006) 1, arXiv: 0501034 [hep-ph].

[4] A. Rybarska, W. Schäfer and A. Szczurek, Phys. Lett. B668 (2008) 126, arXiv: 0805.0717 [hep-ph].

[5] LHCb collaboration, R. Aaij et al., J. Phys. G40 (2013) 045001, arXiv:1301.7084 [hep-ex]

[6] LHCb collaboration, R. Aaij et al., arXiV:1401.3288 [hep-ex].

[7] H1 collaboration, A. Aktas et al., Eur. Phys. J. C46 (2006) 585, arXiv:0510016 [hep-ex].

[8] H1 collaboration, C. Alexa et al., Eur. Phys. J. C73 (2013) 2466. arXiv:1304.5162 [hep-ex].

[9] TOTEM collaboration, G. Antchev et al., Eur. Phys. Lett. 101 (2013) 21002. 